\title{
XXIV.
}

\section{Ueber den Einfluss der Nahrung auf die quantitative Zusammensetzung der Milch.}

\author{
Von Dr. Ssubotin aus St. Petersburg.
}

Es

wird allgemein angenommen, dass die Nabrung einen grossen Einfluss auf die quantitative Zusammensetzung der Milch habe. Aber die Resultate aus vielen Analysen, welche in dieser Beziehung gemacht wurden, widersprechen einander. Fast alle Untersuchungen über diesen Gegenstand wurden an der Kuh und anderen Pflanzenfressern angestellt. Einige Untersuchungen über die Milch der Frauen wurden von Vernois und Becquerel*) gemacht. Aus diesen folgt, dass die Art der Nahrung sehr wenig Einfluss auf die Zusammensetzung der Milch hat, viel wichtiger aber die Menge derselben ist. Ueberflüssige Nahrung vermehrt die tägliche Menge der Milch, sowie die des Casein und der Butter in derselben. Spärliche Nahrung hat den entgegengesetzten Einfluss. Boussingault und la $\mathrm{Bel}{ }^{*}$ ) haben auch für die Kub dasselbe gefunden.

Andere Forscher behaupten, dass die Art der Nahrung die quantitative Zusammensetzung der Milch sehr bedeutend verändere. Thomson ${ }^{* * *}$ ) schliesst aus seinen Untersuchungen, dass Erhöhung des Stickstoffgehalts der Nahrung die Menge der Butter in der Milch erhöhe. Playfair fand umgekehrt, dass bei stickstoffarmer Nahrung die Milch fettreicher werỏe.

Was die Zusammensetzung der Milch der Omnivoren betrifft, io geht aus den Versuchen von Benscht) an Hunden hervor: lass die Menge des Casein bei Fleischnahrung vermehrt, bei vegeabilischer vermindert wird; in letzterem Falle aber der Gehalt an

*) Anvales d'hygiène publique. April 1857. p. 294-296.

**) Ano. de chim. et de phys. Octob. 1844. p. 153.

***) Anv. der Chem. und Pharm. Bd. LXI. S. 242.

†) Liebig's Ann. Bd. 61. S. 221.

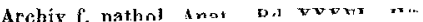


Butter und Zucker vermehrt wird; welcher letztere bei Fleischnahrung nicht ganz versehwindet, aber doch auf ein Minimum reducirt wird.

Solche Widersprüche in einer so wichtigen Erage haben mir Veranlassung zu Untersuchungen im Laboratorium des Hrn. Prof. Pflüger über die Zusammensetzung der Milch bei verschiedener Nahrung gegeben. Meine Untersuchungen wurden an Hündinnen angestellt, welche als Omnivoren mir zu diesen Versuchen am brauchbarsten schienen.

Die Hündinnen, welche ich benutzte, bekamen immer zu derselben Stunde ihe Futter; die Abzapfung der Milch geschah um 11 Uhr Morgens. Vor jeder Milchabzapfung wurden den Thieren einige Stunden (bei allen Versuchen gleich lang) die Jungen weggenommen.

Es gibt viele Methoden der Milchanalyse. Ich gebrauchte davon diejenigen, welche die genauesten Resultate liefern. Zur Bestimmung des Caseingehaltes wurde eine genau gewogene Menge Milch in einem hinreichend hohen Becherglase auf ihr 20 faches Volum mit destillirtem Wasser verdünnt, und unter Umrühren sehr verdünnte Essigsäure tropíenweise so lange binzugefügt, bis sich ein flockiger Niedersehlag zu zeigen begann, nun wurde durch die Flüssigkeit $\frac{1}{4}-\frac{1}{2}$ Stunde lang ein Strom von Kohlensäure geleitet und dann einige Stunden bis Tage bedeckt stehen gelassen. Das Casein schlug sich zusammen mit der Butter nieder, die Flüssigkeit wurde klar, durchsichtig und leicht filtrirbar. Der Niederschlag wurde auf ein gewogenes Filter gesammelt, ausgewascben, getrocknet und gewogen. Die klar abfiltrirte Flüssigkeit wurde dann zum Kochen erhitzt, das sich abscheidende Albumin auf ein gewogenes Filter gesammelt, ausgewaschen, bei $110^{\circ} \mathrm{C}$. getrocknet und gewogen. In der gesammelten vom Albumin abfiltrirten Flüssigkeit wurde der Zueker durch die Fehling'sche Methode bestimmt. Zur Bestimmung der Gesammtmenge der festen Bestandtheile und des Fettes wurde immer die Methode von Haidlen gebraucht.

Gleich nach der Entbindung ist die Milch ron Hündinnen schleimig, klebrig, von weissgelber Farbe. Nach einer Woche verschwindet die Schleimigkeit und die Milch bekommt ihre gewôhnliche Qualität. Die Reaction der Milch war in allen von mir unter. suchten Fällen sauer. 
1. Versuch. Grosse gesunde Hühnerhündin in der dritten Woche nach der Entbindung mit einem Jungen. Ich untersuchte die Milch gleich als ich das Thier erbielt, welches bis dahin wahrscheinlich von-gemischter Kost gelebt hatte.

Auf 1000 Theile Milch fand ich:

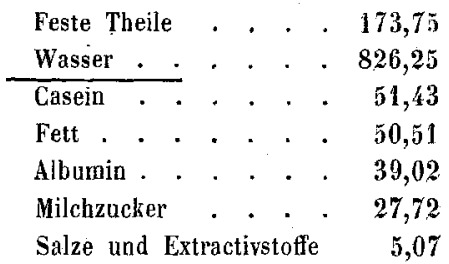

Hierauf wurde sie 8 Tage lang mit täglich 3-4 Pfund Pferdefleisch gefüttert, welches möglichst fettlos war. Nach dieser Zeit waren die Milchdrüsen dick und secernirten reichlich, so dass ich ohne Mühe $60 \mathrm{Ccm}$. Milch zur Analyse erhielt, während ich vorher mit Mühe nur $15 \mathrm{Ccm}$. bekam. Die Hündin war in beiden Fällen ğłeiche Zeit (10 Stunden) ohne Junge.

Auf 1000 Theile Milch fand ich:

\begin{tabular}{|c|c|c|}
\hline Feste Theile & . & 222,10 \\
\hline Wasser . & . . & . $\quad 777,90$ \\
\hline Casein und Fett & . & . $\quad 160,45$ \\
\hline Casein & . & 40,41 \\
\hline Fett. . . & . . & 120,04 \\
\hline Albumin. & . & 32,11 \\
\hline Milehzucker & . . & $2 \tilde{5}, 29$ \\
\hline Salze and Extr & tirut & 4,25 \\
\hline
\end{tabular}

Hierauf wurde die Hündin 4 Tage lang mit einem Gemisch von gekochten Kartoffeln und Stärkemebl gefüttert, welches sie gern frass; sie erhielt soviel sie wünschte. Nach dieser Zeit fand ich die Drüsen zusammengeschrumpft, sie ent* bielten viel weniger Milch als vorber.

Ich fand jetzt auf 1000 Th. Milch:

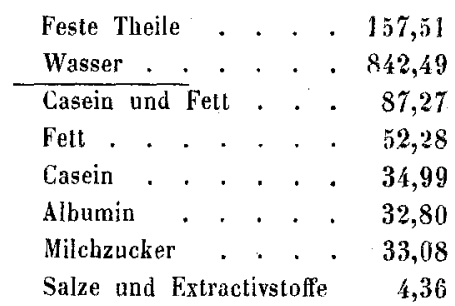

Hierauf wurde dieselbe Fütterung noch 2 Tage fortgesetzt, nach dieser Zeit war die Milch fast ganz geschwuaden und nar mit grosser Mübe erhielt ich, nachdem das Thier 18 Stunden ohne Junge war, 16 Grm. Milch.

Diese enthielt auf 1000

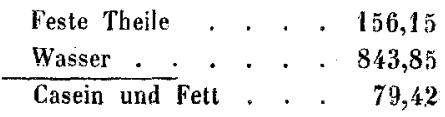




\begin{tabular}{|c|c|c|}
\hline Casein. & . . & . . \\
\hline Fett. & . & $=$ \\
\hline Albumin & - & . \\
\hline Milchzucker & & . . . \\
\hline
\end{tabular}

Nun erbielt die Hündin wieder 3 Tage Fleisch, wodurch die Milch bedeutend vermehrt wurde.

In dieser fanden sich auf 1000

\begin{tabular}{|c|c|c|c|c|}
\hline Feste Theile & & . & & 211,82 \\
\hline Wasser & & . & & 788,18 \\
\hline Casein und & Eett & . & & 145,84 \\
\hline Casein & & . & & 49,76 \\
\hline Fett . & & & & 96,0 \\
\hline Albumin & . & • & & 37,0 \\
\hline Milchzucker & & . & & 25,7 \\
\hline alze und & & & & 3,2 \\
\hline
\end{tabular}

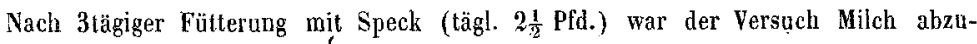
zapfen vergebens. Ich liess die Hündin 18 Stunden ohne Junge und erbielt nur so wenig Milch, dass sich keine zuverlässigen Analysen anstellen liessen. Das Thier war übrigens gesund, munter und zeigte auch keine Verdauungsstörungen.

Als hierauf wieder 2 Tage mit. Fleisch gefüttert war, erschien die Milch in zur Untersuchung hinreichender Menge.

Nachdem nun 2 Tage mit einem Gemisch von Fleisch und Fett (von jedem $1 \frac{1}{2}$ Pfund) gefüttert war, fand ich die Milchmenge so gering, dass ich nur mit Mübe $8 \mathrm{Grm}$. zur Analyse erhalten konnte. Bei dieser geringen Quantität war es nicht möglich, einen Theil besonders zur Bestimmung des Fettes zu benutzen, und desshalb wurde bei diesem Versuch allein das Fett bestimmt durch Extrahiren des auf einem Filter getrockneten Gemisches von Casein und Fett mit Aether und vorund nachberiges Wägen. Die Fehler können hierbei bis $\frac{1}{2}$ pCt. betragen.

Auf 1000 Theile wurden gefunden:

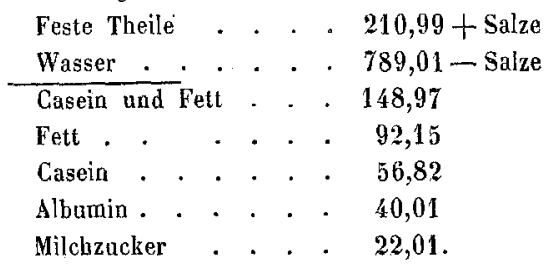

2. Versuch. Grosse Hündin derselben Art mit einem Jungen in der ersten Woche nach der Entbindung. Sie hatte sehr wenig schleimige Milch. Nach 7 Tagen Fleischfütterung war es schon möglich, eine hinreichende Menge Milch zu Analysen zu bekommen.

Es wurden gefunden auf 1000 Theile:

$$
\begin{aligned}
& \text { Feste Theile . . . } \mathbf{2 4 9 , 9 8} \\
& \text { Wasser . . . . . 750,02 } \\
& \text { Fett und Casein . , 161,11 }
\end{aligned}
$$




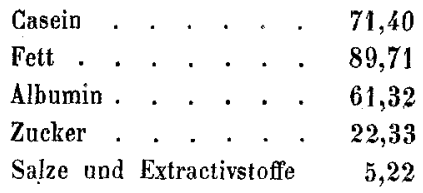

Durch 3tägige Fütterung mit Kartoffeln und Stärkemebl wurde die Milch vermindert.

Die Untersuchung zeigte auf 1000 Theile:

\begin{tabular}{|c|c|c|c|c|}
\hline Feste Theile & & & . & . 194,49 \\
\hline Wasser. & & $\cdot$ & $\cdot$ & . 805,51 \\
\hline Casein und & Fett & & · & 100,72 \\
\hline Cásein & & . & 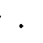 & 48,05 \\
\hline Fett & . & . & 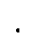 & 52,67 \\
\hline Albumin & . & - & . & 51,41 \\
\hline Milchzucker & . & . & · & 37,29 \\
\hline alze und & $\mathrm{F}$ & & & 5, \\
\hline
\end{tabular}

Nachdem nun 7 Tage mit Fleisch gefüttert worden, fand sich viel mebr Milch und die Analyse ergab auf 1000 Theile:

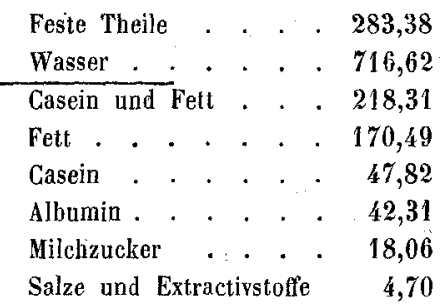

Nach 2 Tagen Fütterung mit Speck konnte ich keine Milch zur Analyse bekommen, sie war beinahe ganz geschwunden. Das Thier war gesund, keine Störung der Verdauung.

3. Versuch. Grosser Pudel mit 3 Kleinen in der dritten Woche nach der Entbindung. Die Dräsen waren sebr gross und lieferten ausserordentlich viel Milch, dieser Hund wurde vor abzapfung der Mileh nur 3 Stunden ohne Kleine gelassen.

Nach 1 Tag Fütterung mit Flejsch (2 Pfund) wurden gefunden auf 1000 Theiler Milch:

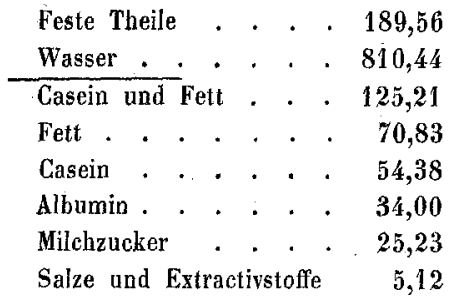

Nach 2 Tagen Fütterung mit Speck (täglich $1 \frac{1}{2}$ Pfund), welchen sie ungern 'ass, waren die Drüsen zusammengeschrumpft, die Milch sehr vermindert, so dass ih nur mit Mühe $25 \mathrm{Grm}$. zur Analyse erhielt, während ich bei dem vorigen Versche mit Leichtigkeit 75 Grm. erhielt. 


\section{6}

Die Milch enthielt auf 1000 Theile:

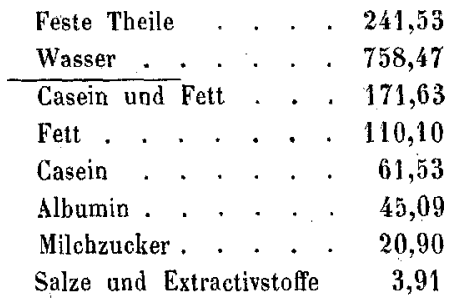

Der Hund wurde nun 2 Tage mit Fleisch gefüttert, Mileh erschien wieder in Ueberffuss. Dieselbe enthielt auf 1000 Theile:

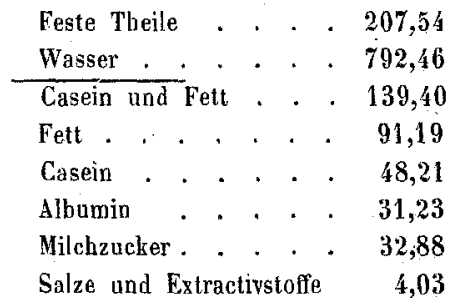

Nach einem Tage ohne Nahrung wurde in der Drïse viel mehr Milch, als bei Fettfïtterung gefunden.

In 1000 Theilen waren:

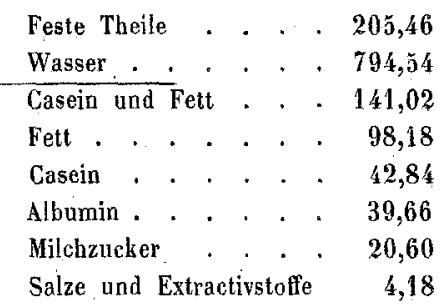

Alle vorhergehenden Versuche habe ich der bequemeren Uebersieht wegen in eine Tabelle zusammengestellt und dabei zugleich die mittleren Werthe aus denselben angegeben. 


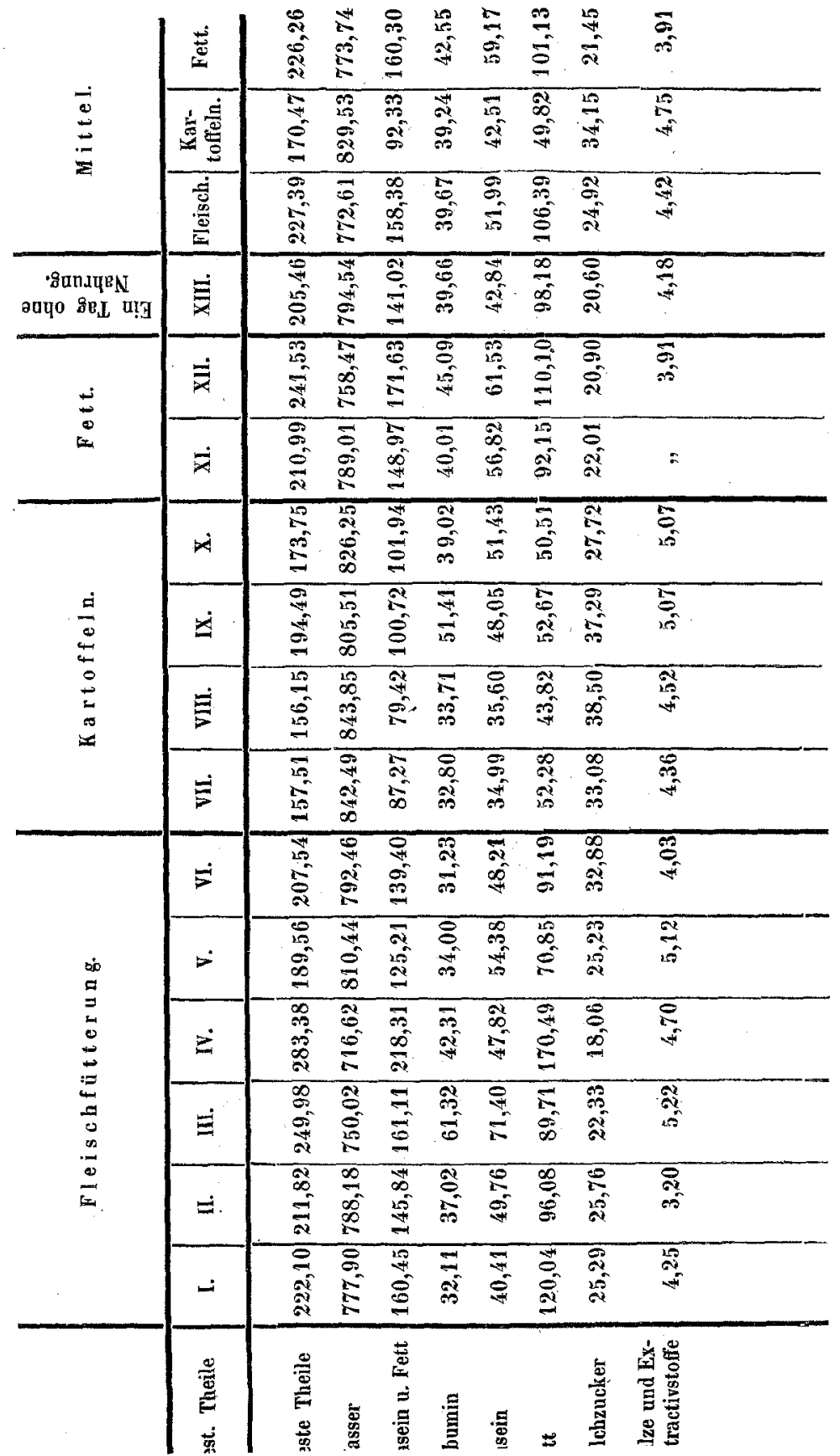


Bei Uebersicht dieser Resultate finden wir im Gegensatze zu vielen früheren Forschern, dass die Art der Nahrung bedeutenden Einfluss auf die quantitative Zusammensetzung der Milch hat. Die Menge der festen Bestandtheile nimmt bei animaler Kost zu und diese Vermehrung fällt hauptsächlich auf die Fette (bei Fleischfütterung 106, bei Kartoffeln 49 auf 1000). Die Vermehrung des Casein ist weniger bedeutend $(1 \mathrm{pCt}$.). Diese beiden Bestandtheile nehmen ubrigens nicht nur relativ, sondern auch absolut zu, da die täglich secernirte Milchmenge bei animaler Nahrung grösser ist. Der Gehalt an Albumin und Salzen bleibt beinahe unverändert. Zucker findet sich bei Hunden bei vegetabilischer Kost bis $3 \frac{1}{2} \mathrm{pCt}$., bei Fleischfütterung beobachtete ich nie, bei meinen sebr sorgfältigen Analysen, ein Herabsinken des Zuckergehaltes auf ein Minimum, wie Benseh behauptet; derselbe fiel nur auf 2,4 pCt., so dass die Differenz gegen vegetabilische Nahrung nur $1 \mathrm{pCt}$. betrug. Die Meinung von Bensch, Playfair und Anderen, dass bei vegetabilischer Kost sich der Gehalt an Fett vermehrt, bei animaler sich vermindert, ist nicht richtig; keine der von mir gemachten Analysen spricht dafür. Beim Uebergange von animaler zu vegetabilischer Nahrung vermindert sich die Menge der festen Bestandtheile, namentlich Fett und Casein, während Zucker nur unbedeutend zunimmt.

Bei Fettfütterung vermehren sich jedoch nur relativ die festen Bestandtheile, besonders Fett, bei gleichzeitiger Verminderung des Zuckers.

Genaue Bestimmungen der täglichen Milchmenge sind bei Hunden nicht möglich. Meinen Beobachtungen nach sind bei Fleischfülterung die Drüsen dick und die Milch spritzt beim Drücken auf dieselben in einem Strahle hervor, man kann leicht durch Abzapfung zu Analysen 40-100 Ccm. bekommen. Bei vegetabilischer Kost sind die Drüsen welk und man erhält nur mit grosser Mühe $\frac{1}{2}-\frac{1}{3}$ der eben angegebenen Menge. In beiden Fällen waren die Thiere, abgesehen von der Nahrung, unter gleichen Bedingungen; erhielten ihr Futter zur selben Zeit, waren gleichlang ohne Junge etc. Wurde von vegetabilischer zu Fleischnahrung übergegangen, so erschien nach 1-2 Tagen Milch wieder in grosser Menge.

Besonders interessant ist die auffallende Verminderung der Menge der Milch bei Fettfütterung bis zu gänzlichem Verschwinden 
derselben. Bei zwei Hunden war nach einigen Tagen Fütterung mit Fett die Milch gänzlich verschwunden und erschien wieder nach Fütterung mit Fleisch. Sogar bedeutender Zusatz von Fett zur Nahrung ( $1 \frac{1}{2} \mathrm{Pfd}$. Fleisch und $1 \frac{1}{2} \mathrm{Pfd}$. Fett) vermindert die Milch so sehr, dass ich nach 3tägiger Fütterung mit diesem Gemisch keine zur Analyse hinreichende Menge erhalten konnte, während der Hund vorher sebr viel Milch lieferte.

Die von mir gefundene Thatsache, dass Fleischfütterung die Menge der Butter in der Milch so sehr vermehrt, zeigt, dass das Fett der Milch wenigstens zum grossen Theil aus Albumin gebildet wird; denn die Meinung ron Boussingault, dass wenn die Nahrung wenig Fett enthält, die Butter auf Kosten des Fettes des Körpers gebildet wird, wird unter anderen auch schon dadurch widerlegt, dass die von mir benutzten Hunde, welche anfangs mager waren, während der Fütterung mit Fleisch fett wurden. Das Fett häufte sich in bedeutenden Massen im Unterhautbindegewebe, im Netz, der Nierenkapsel und zwischen den Muskelbündeln an. Diese Beobachtung ist meiner Meinung nach ein Beweis für die Unwandlung von Albumin in Fett innerhalb des Organismus uberhaupt.

Unter den wenigen Beobachtungen, die für diese Umwandlung sprechen, sind die beweisendsten die von Burdach und Hoppe. Ersterer wies nach, dass bei der Entwickelung der Eier von Limnaeus stagnalis die Menge des Fettes vermehrt, die des Albumin vermindert wird. Der Werth dieser Untersuchungen wird aber dadurch herabgesetzt, dass dieselben sich auf 2 Analysen beschränken. Hoppe*) wies nach, dass, wenn man Milch an der Luft stehen lässt, sich die Menge der Butter vermehrt. Diese Vermehrung ist freilich so gering, dass sie innerhalb der Fehler der Analyse fällt, und nur aus ihrer Constanz (6mal unter 7 Fällen) lässt sich auf die Richtigkeit der Beobachtung schliessen. Es versteht sich, dass diese Beobachtungen von Hoppe an Beweiskraft gewinnen, wenn sie in grösserem Umfange wiederholt werden. Ich habe 10 Analysen nach derselben Methode, wie Hoppe gemacht.

In der ersten Columne ist der procentische Fettgehalt der frischen Milch, in der zweiten derselbe nachdem die Milch eine bestimmte Zeit gestanden, in der dritten diese Zeit in Stunden angegeben.

*) Dieses Archiv Bd, XVII. 


$\begin{array}{rccc} & \text { I. } & \text { II. } & \text { III. } \\ \text { 1) } & 4,72 & 4,85 & \mathbf{2 4} \\ \text { 2) } & 3,17 & 3,49 & 96 \\ \text { 3) } & 1,99 & 2,27 & 96 \\ 4) & 6,25 & 6,29 & 96 \\ \text { 5) } & 3,14 & \mathbf{3 , 5 6} & 48 \\ 6) & 4,11 & 4,30 & 96 \\ 7) & 4,82 & 5,63 & 36 \\ 8) & 1,88 & 1,90 & 96 \\ 9) & 3,50 & 3,42 & 96 \\ \text { 10) } & 4,04 & 4,28 & 96\end{array}$

Diese Analysen sprechen auch für die vorher ausgesprochene Meinung, dass die Albuminate in der Milch in Fett ubergehen.

\section{XXV.}

Wissenschaft und Charlatanerie unter den Arabern im neunten Jalirhundert.

Nach der hebräischen Uebersetzung eines Schriftchens von Rhases, mitgetheilt von M. Steinschneider.

Vorbemerkung des deutschen Bearbeiters.

Der Kampf der ernsten Wissenschaft mit einem, nach dem Scheine strebenden trügerischen Gewerbe ist in seinen wesentlichen Argumenten wohl überall und jederzeit derselbe, wie auch die besonderen Thatsachen und Motive nach Zeiten und Orten, nach Culturstufen und Arten der Wissenschaft sich von einander unterscheiden. Darum wird auch dieser Kampf schwerlich je überflüssig werden: wenn die Wissenschaft sich abmüht, das Gebiet der Wahrheit und Erkenntniss zu erweitern, so ist es der Täuschung ein Leichtes, neue Mittel und Wege nicht nur zum grossen Haufen zu finden, sondern auch Männer des Gedankens wenigstens zu verdutzen, ihnen den Muth eines entschiedenen Protestes zu rauben, oder ihre Zeit und Kraft für Prüfung und Bestreitung des stets neu auftauchenden Wahnes in Anspruch zu nehmen. 